Funding: This work was undertaken while MJ was visiting scholar to the department of family practice, University of British Columbia, Canada. He was funded by the department of primary care and population sciences, Royal Free and University College Medical Schools, University College London, NHS prolonged study leave, and a grant from the King's Fund. Competing interests: None declared.

1 Department of Health. NHS primary care walk in centres. London: $\mathrm{DoH}$, 1999 (HSC 1999/116)

2 Wilkie P, Logan A. Walk in centres. Br J Gen Pract 2000;49:1017.

3 Shah CP. Public health and preventive medicine in Canada. Toronto: University of Toronto Press, 1994.

4 Feldman W, Cullum C. The pediatric walk-in clinic. CMAJ 1984;130:1003-5.

5 Miller GB, Mah Z, Nantes S, Bryant W, Kayler T, McKinnon K. Ontario walk in clinics. Can Fam Physician 1989;35:2013-5.

6 Rizos J, Anglin P, Grava-Gubins I, Lazar C. Walk-in clinics. CMAJ 1990;143:740-5.

7 Bell NR, Szafran O. Use of walk in clinics by family practice patients. Can Fam Physician 1992;38:507-13.

8 Rachlis V. Who goes to after-hours clinics? Can Fam Physician 1993;39:226-70.
9 Burnett MG, Grover SA. Use of the emergency department for non-urgent care. CMAJ 1996;154:1345-51.

10 Grad R, Kaczorowski J, Singer Y, Levitt C, Mandelcorn J. Where do family practice patients go in case of emergency? Can Fam Physician 1998:44:2666-72.

11 Weinkauf DJ, Kralj B. Medical service provision and costs. Can Public Policy 1998;24:471-84

12 Szafran O, Bell NR. Use of walk in clinics by rural and urban patients. Can Fam Physician 2000;46:114-9.

13 Miller GB, Nantes S. Walk in clinics and primary care. Can Fam Physician 1989:35:2019-22.

14 Borkenhagen RH. Walk in clinics. Can Fam Physician 1996;42:1879-83.

15 Milne P. Could you compete with a walk-in clinic? CMAJ 1987;136:534.

16 What's wrong with walk-ins? CMAJ 1988;139:63-4.

17 Burak AJ. Walk in clinics. Br Columbia Coll Fam Physicians 1994; October:5-6.

18 Makin MD. McDonald's medicine. Br Columbia Med J 1993;35:151

19 Milne M. Walk-in clinics. CMAJ 1987;137:532-6.

20 Rizos J. Walk-in clinics. CMAJ 1991;144:631-2.

21 Rowlands J. After hours clinics. Ont Med Rev 1988;55:10-7

22 Wishart D. BC GPs unite to fight walk in clinics. CMAJ 1987;137:535.

23 Toews B. Walk in clinics add to costs. Br Columbia Med J 1992;34:202.

(Accepted 10 July 2000)

\title{
Effect of needle length on incidence of local reactions to routine immunisation in infants aged 4 months: randomised controlled trial
}

\author{
Linda Diggle, Jonathan Deeks
}

\begin{abstract}
Objective To compare rates of local reactions associated with two needle sizes used to administer routine immunisations to infants.

Design Randomised controlled trial.

Setting Routine immunisation clinics in eight general practices in Buckinghamshire.

Participants Healthy infants attending for third primary immunisation due at 16 weeks of age: 119 infants were recruited, and 110 diary cards were analysed.

Interventions Immunisation with 25 gauge, $16 \mathrm{~mm}$, orange hub needle or 23 gauge, $25 \mathrm{~mm}$, blue hub needle.
\end{abstract}

Main outcome measures Parental recordings of redness, swelling, and tenderness for three days after immunisation.

Results Rate of redness with the longer needle was initially two thirds the rate with the smaller needle (relative risk 0.66 (95\% confidence interval 0.45 to $0.99), \mathrm{P}=0.04)$, and by the third day this had decreased to a seventh (relative risk 0.13 (0.03 to $0.56), P=0.0006)$. Rate of swelling with the longer needle was initially about a third that with the smaller needle (relative risk 0.39 (0.23 to 0.67$), \mathrm{P}=0.0002$ ), and this difference remained for all three days. Rates of tenderness were also lower with the longer needle throughout follow up, but not significantly (relative risk 0.60 (0.29 to 1.25$), \mathrm{P}=0.17$ ).

Conclusions Use of $25 \mathrm{~mm}$ needles significantly reduced rates of local reaction to routine infant immunisation. On average, for every five infants vaccinated, use of the longer needle instead of the shorter needle would prevent one infant from experiencing any local reaction. Vaccine

manufacturers should review their policy of supplying the shorter needle in vaccine packs.

\section{Introduction}

As part of the UK childhood immunisation schedule, infants routinely receive diphtheria, pertussis, and tetanus (DPT) vaccine and Haemophilus influenzae type b (Hib) vaccine at 2, 3, and 4 months. ${ }^{1}$ Nationally available guidelines advise practitioners to administer primary vaccines to infants by deep subcutaneous or intramuscular injection using either a 25 or 23 gauge needle but give no recommendation regarding needle length. ${ }^{1}$ The question of optimum needle length for infant immunisation has not previously been addressed in Britain, despite calls from nurses for evidence on which to base immunisation practice. We conducted a randomised controlled trial of the two needle sizes currently used by UK practitioners to determine whether needle size affects the incidence of redness, swelling, and tenderness.

\section{Participants and methods}

\section{Participants}

Eight of 11 general practices approached in Buckinghamshire agreed to participate in the study. Practice nurses recruited healthy infants attending routine immunisation clinics. Parents received written information about the study when attending for the second primary vaccination and were asked if they wished to participate when they returned for the third vaccination. The only exclusion criteria were those normally applicable to a child receiving primary immunisations.
Oxford Vaccine
Group, University
Department of
Paediatrics, John
Radcliffe Hospital,
Oxford OX3 9DU
Linda Diggle
senior research nurse 


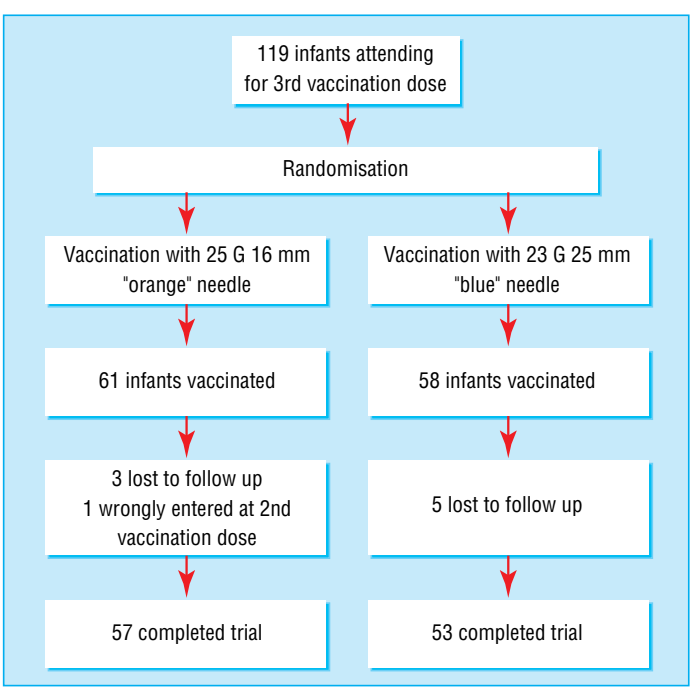

Flow chart describing randomisation sequence

We obtained ethical approval from the local ethics committee.

\section{Interventions}

Infants were allocated to receive their third primary immunisation with either the 25 gauge, $16 \mathrm{~mm}$ needle or the 23 gauge, $25 \mathrm{~mm}$ needle according to a computer generated blocked randomisation scheme stratified by practice. Allocations were concealed in sequentially numbered opaque envelopes opened once written parental consent was obtained. Practice nurses were instructed verbally, by demonstration and in writing, to use the technique of injecting into the anterolateral thigh, stretching the skin taut and inserting the needle at a $90^{\circ}$ angle to the skin. ${ }^{2}$ The right thigh was used, with the needle inserted into the skin up to the hub.

\section{Outcomes}

Parents recorded redness, swelling, and tenderness in a diary for three days after immunisation. The size of swelling and redness were measured with a plastic ruler, while the child's reaction to movement of the limb or to touch of the site was graded with a standard scale. We supplied parents with a prepaid envelope to return the diary, and we contacted parents by telephone if return was delayed.

At the start of the trial all practices were using the $0.5 \mathrm{ml}$ mix of Pasteur-Merieux DPT/Hib vaccine. However, a change in national vaccine supply necessitated a switch to the $1.0 \mathrm{ml}$ mix of Evans DPT and Wyeth Lederle Hib-Titer. Blocked randomisation ensured that the numbers receiving each vaccine were evenly distributed between the groups.

\section{Statistical analysis}

In order to detect clinically important relative differences of $25 \%$ in tenderness and $30 \%$ in redness

Baseline characteristics of 4 month old infants and rate of local reactions to immunisation over three days by needle used for vaccination. Values are numbers (percentages) of infants unless stated otherwise

\begin{tabular}{|c|c|c|c|c|}
\hline \multirow[b]{2}{*}{ Local reaction } & \multicolumn{2}{|c|}{ Size of needle } & \multicolumn{2}{|c|}{ Difference between longer and shorter needle } \\
\hline & $23 \mathrm{G}, 25 \mathrm{~mm}(\mathrm{n}=53)$ & $25 \mathrm{G}, 16 \mathrm{~mm}(\mathrm{n}=57)$ & Relative risk ( $95 \% \mathrm{CI}) ; \mathrm{P}$ value & Test for trend \\
\hline \multicolumn{5}{|l|}{ Baseline characteristics } \\
\hline Mean (SD) weight $(\mathrm{kg})^{*}$ & $6.7(0.9)$ & $6.8(0.9)$ & & \\
\hline \multicolumn{5}{|l|}{ Age at vaccination (weeks): } \\
\hline $16-17$ & $37(70)$ & $36(63)$ & & \\
\hline $18-19$ & $11(21)$ & $16(28)$ & & \\
\hline$\geqslant 20$ & $5(9)$ & $5(9)$ & & \\
\hline \multicolumn{5}{|l|}{ Sex } \\
\hline Male & $34(64)$ & $30(53)$ & & \\
\hline Female & $19(36)$ & $27(47)$ & & \\
\hline \multicolumn{5}{|l|}{ Site of injection: } \\
\hline Left leg & $13(25)$ & $12(21)$ & & \\
\hline Right leg & $40(75)$ & $45(79)$ & & \\
\hline \multicolumn{5}{|l|}{ Vaccine type†: } \\
\hline $0.5 \mathrm{ml}$ & $8(15)$ & $8(14)$ & & \\
\hline $1.0 \mathrm{ml}$ & $45(85)$ & $49(86)$ & & \\
\hline \multicolumn{5}{|l|}{ Local reactions } \\
\hline \multicolumn{5}{|l|}{ Redness: } \\
\hline At 6 hours & $21(40)$ & $34(60)$ & 0.66 (0.45 to 0.99$) ; P=0.04$ & $P=0.007$ \\
\hline At 1 day & $15(28)$ & $36(63)$ & 0.45 (0.28 to 0.72$) ; P=0.0002$ & $P<0.0001$ \\
\hline At 2 days & $5(9)$ & $22(39)$ & 0.24 (0.10 to 0.60$) ; P=0.0004$ & $P=0.0004$ \\
\hline At 3 days & $2(4)$ & $16(28)$ & 0.13 (0.03 to 0.56 ); $P=0.0006$ & $P=0.001$ \\
\hline \multicolumn{5}{|l|}{ Swelling: } \\
\hline At 6 hours & $12(23)$ & $33(58)$ & 0.39 (0.23 to 0.67$) ; P=0.0002$ & $P=0.0009$ \\
\hline At 1 day & $15(28)$ & $36(63)$ & 0.45 (0.28 to 0.72 ); $P=0.0002$ & $\mathrm{P}=0.0001$ \\
\hline At 2 days & $10(19)$ & $29(51)$ & 0.37 (0.20 to 0.69 ); $P=0.0005$ & $P=0.0007$ \\
\hline At 3 days & $7(13)$ & $23(40)$ & 0.33 (0.15 to 0.70$) ; P=0.001$ & $P=0.002$ \\
\hline \multicolumn{5}{|l|}{ Tenderness: } \\
\hline At 6 hours & $9(17)$ & $16(28)$ & 0.60 (0.29 to 1.25$) ; P=0.17$ & $P=0.4$ \\
\hline At 1 day & $4(8)$ & $8(14)$ & 0.54 (0.17 to 1.68$) ; P=0.3$ & $P=0.4$ \\
\hline At 2 days & 0 & $3(5)$ & 0 (not estimable); $P=0.09$ & $P=0.4$ \\
\hline At 3 days & 0 & $1(2)$ & 0 (not estimable); $P=0.3$ & $\mathrm{P}=0.2$ \\
\hline Any local reaction & $33(62)$ & $48(84)$ & 0.74 (0.58 to 0.94 ); $P=0.009$ & \\
\hline
\end{tabular}

*Weight missing for three infants.

†0.5 ml vaccine=Pasteur Merieux DPT/Hib. $1 \mathrm{ml}$ vaccine=Evans DPT reconstituting Wyeth Lederle Hib-Titer. 
and swelling, we estimated that 250 infants should be recruited for the study to have $80 \%$ power of detecting differences at the 5\% significance level. In January 2000 , problems with vaccine supply necessitated the temporary nationwide replacement of the whole cell component of the combined DPT/Hib vaccine with acellular pertussis vaccine. ${ }^{3}$ As this vaccine has a different local reactogenicity profile, we decided to stop the trial early.

We used $\chi^{2}$ tests to compare the proportions of children with each local reaction at 6 hours and 1, 2, and 3 days after immunisation. We compared differences in the size of reaction using a $\chi^{2}$ test for trend.

\section{Results}

Of the 119 children recruited to the study, 61 were randomised to the $16 \mathrm{~mm}$ needle group and 58 to the $25 \mathrm{~mm}$ needle group (see figure). Nine were not included in the analysis (four in the $16 \mathrm{~mm}$ needle group and five in the $25 \mathrm{~mm}$ group): diaries were not returned for eight, while the ninth was mistakenly included in the study at the second vaccination. Inclusion of this child did not materially affect the results. The two groups had similar baseline characteristics (see table).

Over half of the infants vaccinated with the $16 \mathrm{~mm}$ needle subsequently experienced redness and swelling (table). The rate of redness with the $25 \mathrm{~mm}$ needle was initially two thirds the rate with the $16 \mathrm{~mm}$ needle (relative risk 0.66 (95\% confidence interval 0.45 to $0.99)$ ), and, by the third day, this had decreased further to a seventh (relative risk 0.13 (0.03 to 0.56$)$ ). Similarly, rates of swelling after injection with the longer needle were initially around a third of those after use of the smaller needle (relative risk 0.39 (0.23 to 0.67)), and this difference was maintained for all three days. These differences were statistically significant. Tenderness was less frequent and, although the rates of tenderness were also lower with the longer needle throughout follow up, the differences were not significant (table).

\section{Discussion}

This study showed that both redness and swelling were significantly reduced when the 23 gauge, $25 \mathrm{~mm}$, blue hub needle was used instead of the 25 gauge, $16 \mathrm{~mm}$, orange hub needle to administer the third dose of diphtheria, pertussis, and tetanus and Haemophilus influenzae type b vaccines to infants. The differences suggest that, for every three to five infants vaccinated with the longer rather than the shorter needle, one case of redness and one of swelling would be prevented.

The needles compared in this study are those most commonly used in general practice. ${ }^{4}$ As they differed in both length (16 $v 25 \mathrm{~mm}$ ) and bore (25 $v 23$ gauge), we cannot know which of these factors determined the observed differences in the rates of redness and swelling. However, previous studies comparing injections given at different depths (subcutaneous versus intramuscular) with the same gauge needle have shown similar differences in local reactions. ${ }^{56}$ We suggest that the length of the longer needle used in our
What is already known on this topic

Most infants experience local reactions to routine vaccinations

Previous local reactions have been cited by parents as a disincentive to further vaccinations

National guidelines on immunisation do not specify a preferred needle length

\section{What this study adds}

Local reactions are significantly reduced by use of the 23 gauge, $25 \mathrm{~mm}$, blue hub needle rather than the 25 gauge, $16 \mathrm{~mm}$, orange hub needle supplied by vaccine manufacturers

study ensured that the vaccine reached the thigh muscle in 4 month old infants.

Although our study was not blinded, parents were not told which needle was used to vaccinate their child. We believe that if knowledge of needle allocation introduced bias into the results, it would be less likely that such bias would be in the direction of the longer needle.

These findings are of clinical importance for those involved in administering infant immunisations. In the United Kingdom, where routine vaccines are currently supplied with the shorter needle, a change in the manufacturing process is now required. Any factor that can reduce the rates of adverse reactions in childhood vaccinations has the potential to improve parental acceptance of vaccines ${ }^{7}$ and would be welcomed by practitioners.

We thank the parents and babies involved in the study, and the following practice nurses at Buckinghamshire surgeries for recruiting infants and administering immunisations: Lyn Hurry, Waddesdon; Lyn Murphy, Whitehill; Carol Gill, Aston Clinton; Judith Brown, Meadowcroft; Cesca Carter, Wendover; Nicky Oliver, Oakfield; Chris Mildred, Wing; Clare Stroud, Tring Road. We also thank Professor Richard Moxon and Drs Paul Heath, Jim Buttery, Jodie McVernon, Jenny MacLennan, and Karen Sleeman from the Oxford Vaccine Group for helpful advice and support and Dr Ann Mulhall for research supervision.

Contributors: LD conceived and planned the study, recruited and trained practice nurses, managed data collection, wrote the first draft of the paper, and is guarantor for the study.

JD advised on design, produced the randomisation scheme, and undertook all analyses. Both authors had input into the final manuscript.

Funding: This study was funded by the Smith and Nephew Foundation through the award of a nursing research scholarship.

Competing interests: None declared.

1 Department of Health. Immunisation against infectious diseases. London: HMSO, 1996.

2 World Health Organisation. Immunisation in practice. Module 8. During a session: giving immunisations. Geneva: WHO, 1998. (www.who.int/vaccinesdocuments/DoxTrng/H4IIP.htm (accessed 3 October 2000).)

3 Department of Health. Current vaccine issues: action update. London: $\mathrm{DoH}$, 1999. (Professional letter PL/CMO/99/5.)

4 Diggle L. A randomised controlled trial of different needle lengths on the incidence of local reactions when administering the combined injection of diphtheria/pertussis/tetanus (DPT) and Haemophilus influenzae type b (Hib) to infants at 4-months of age [dissertation]. London: Royal College of Nursing Institute, 1999

5 Mark A, Carlsson R, Granstrom M. Subcutaneous versus intramuscula injection for booster DT vaccination of adolescents. Vaccine 1999;17:2067-72.

6 Scheifele DW, Bjornson G, Boraston S. Local adverse effects of meningococcal vaccine. Can Med Assoc J 1994;150:14-5.

7 Lieu T, Black S, Ray G, Martin K, Shinefield H, Weniger B. The hidden costs of infant vaccination. Vaccine 2000;19:33-41.

(Accepted 22 September 2000) 